\title{
OBSERVATION OF COSMIC-RAY ANISOTROPY WITH THE ICETOP AIR SHOWER ARRAY
}

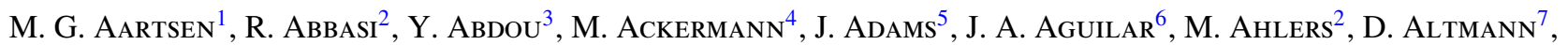

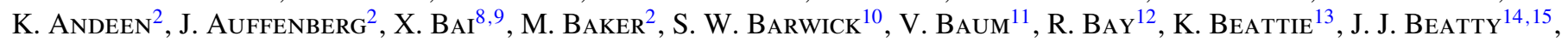
S. Bechet ${ }^{16}$, J. Becker Tuus ${ }^{17}$, K.-H. Becker ${ }^{18}$, M. Bell ${ }^{19}$, M. L. Benabderrahmane ${ }^{4}$, S. BenZvi ${ }^{2}$, J. Berdermann ${ }^{4}$, P. Berghaus ${ }^{4}$, D. Berley ${ }^{20}$, E. Bernardini ${ }^{4}$, D. Bertrand ${ }^{16}$, D. Z. Besson ${ }^{21}$, D. Bindig ${ }^{18}$, M. BissoK ${ }^{22}$, E. Blaufuss ${ }^{20}$, J. Blumenthal ${ }^{22}$, D. J. Boersma ${ }^{22,23}$, S. BohaichuK ${ }^{24}$, C. Bohm ${ }^{25}$, D. Bose ${ }^{26}$, S. BöSer ${ }^{27}$, O. BotNer ${ }^{23}$, L. Brayeur ${ }^{26}$, A. M. Brown ${ }^{5}$, R. Bruijn ${ }^{28}$, J. Brunner ${ }^{4}$, M. Carson ${ }^{3}$, J. CASEy $^{29}$, M. Casier ${ }^{26}$, D. Chirkin ${ }^{2}$, B. Christy ${ }^{20}$, K. Clark ${ }^{19}$, F. Clevermann ${ }^{30}$, S. Cohen ${ }^{28}$, D. F. Cowen ${ }^{19,31}$, A. H. Cruz Silva ${ }^{4}$, M. Danninger ${ }^{25}$, J. Daughhetee ${ }^{29}$, J. C. Davis ${ }^{14}$, C. De Clercq 26 , S. De Ridder ${ }^{3}$, F. Descamps ${ }^{2}$, P. Desiati ${ }^{2}$, G. DE Vries-Uiterweerd ${ }^{3}$, T. DeYoung ${ }^{19}$, J. C. Díaz-VélezZ ${ }^{2}$, J. Dreyer ${ }^{17}$, J. P. Dumm ${ }^{2}$, M. Dunkman ${ }^{19}$, R. EAgan $^{19}$, J. Eisch ${ }^{2}$, R. W. Ellsworth ${ }^{20}$, O. ENGdegÅRD ${ }^{23}$, S. EuleR ${ }^{22}$, P. A. Evenson ${ }^{8}$, O. FAdiran ${ }^{2}$, A. R. FAZely ${ }^{32}$, A. Fedynitch ${ }^{17}$, J. Feintzeig ${ }^{2}$, T. Feusels ${ }^{3}$, K. Filimonov ${ }^{12}$, C. Finley ${ }^{25}$, T. Fischer-Wasels ${ }^{18}$, S. Flis ${ }^{25}$, A. FrancKowiak ${ }^{27}$, R. Franke ${ }^{4}$, K. Frantzen ${ }^{30}$, T. Fuchs ${ }^{30}$, T. K. Gaisser ${ }^{8}$, J. Gallagher ${ }^{33}$, L. Gerhardt ${ }^{12,13}$, L. Gladstone ${ }^{2}$, T. GlüsenKamp ${ }^{4}$, A. Goldschmidt ${ }^{13}$, G. Golup ${ }^{26}$, J. A. Goodman ${ }^{20}$, D. Góra ${ }^{4}$, D. Grant $^{24}$,

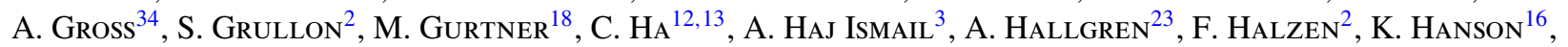

D. Heereman ${ }^{16}$, P. HeimanN ${ }^{22}$, D. Heinen ${ }^{22}$, K. Helbing ${ }^{18}$, R. Hellauer ${ }^{20}$, S. Hickford ${ }^{5}$, G. C. Hill ${ }^{1}$, K. D. Hoffman ${ }^{20}$,

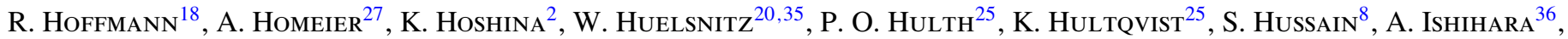

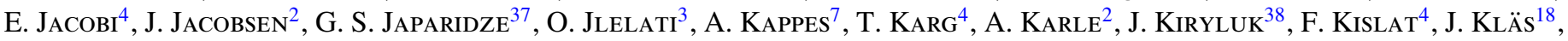

S. R. KLein ${ }^{12,13}$, J.-H. KöHNe ${ }^{30}$, G. Kohnen ${ }^{39}$, H. Kolanoski ${ }^{7}$, L. KÖPKE ${ }^{11}$, C. Kopper ${ }^{2}$, S. Kopper ${ }^{18}$, D. J. KosKINEN ${ }^{19}$,

M. Kowalski ${ }^{27}$, M. Krasberg ${ }^{2}$, G. Kroll ${ }^{11}$, J. Kunnen ${ }^{26}$, N. Kurahashi ${ }^{2}$, T. Kuwabara ${ }^{8}$, M. Labare ${ }^{26}$, H. Landsman ${ }^{2}$, M. J. Larson ${ }^{40}$, R. Lauer ${ }^{4}$, M. Lesiak-Bzdak ${ }^{38}$, J. LÜnemann ${ }^{11}$, J. MadSen ${ }^{41}$, R. Maruyama ${ }^{2}$, K. Mase ${ }^{36}$, H. S. Matis ${ }^{13}$, F. McNally ${ }^{2}$, K. Meagher $^{20}$, M. MercK ${ }^{2}$, P. Mészáros ${ }^{19,31}$, T. Meures ${ }^{16}$, S. Miarecki $^{12,13}$, E. Middell ${ }^{4}$, N. MilkeE ${ }^{30}$, J. Miller ${ }^{26}$, L. Mohrmann ${ }^{4}$, T. Montaruli ${ }^{6,42}$, R. Morse ${ }^{2}$, R. Nahnhauer $^{4}$, U. Naumann ${ }^{18}$, S. C. Nowicki ${ }^{24}$,

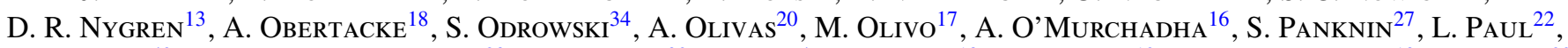
J. A. PepPer ${ }^{40}$, C. Pérez de los Heros $^{23}$, D. Pieloth ${ }^{30}$, N. Pirk ${ }^{4}$, J. Posselt ${ }^{18}$, P. B. Price ${ }^{12}$, G. T. Przybylski ${ }^{13}$, L. RäDel $^{22}$, K. Rawlins ${ }^{43}$, P. RedL ${ }^{20}$, E. Rescon ${ }^{34}$, W. Rhode ${ }^{30}$, M. Ribordy ${ }^{28}$, M. Richman ${ }^{20}$, B. Riedel ${ }^{2}$, J. P. Rodrigues ${ }^{2}$,

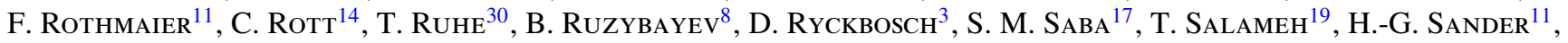
M. SAntander ${ }^{2}$, S. Sarkar ${ }^{44}$, K. Schatto ${ }^{11}$, M. Scheel ${ }^{22}$, F. Scheriad ${ }^{30}$, T. Schmidt ${ }^{20}$, M. Schmitz ${ }^{30}$, S. Schoenen 22 ,

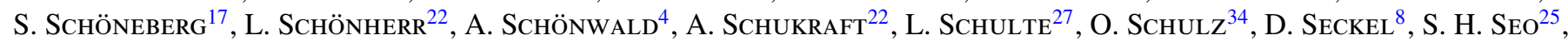
Y. Sestayo ${ }^{34}$, S. Seunarine ${ }^{41}$, C. Sheremata ${ }^{24}$, M. W. E. Smith ${ }^{19}$, M. Soiron ${ }^{22}$, D. Soldin ${ }^{18}$, G. M. SpiczaK ${ }^{41}$, C. Spiering $^{4}$, M. Stamatikos ${ }^{14,45}$, T. Stanev ${ }^{8}$, A. Stasik ${ }^{27}$, T. Stezelberger ${ }^{13}$, R. G. Stokstad ${ }^{13}$, A. Stössl ${ }^{4}$, E. A. Strahler ${ }^{26}$, R. Ström ${ }^{23}$, G. W. Sullivan ${ }^{20}$, H. TaAvola ${ }^{23}$, I. Taboada ${ }^{29}$, A. Tamburro ${ }^{8}$, S. Ter-Antonyan ${ }^{32}$, S. Tilav $^{8}$, P. A. Toale ${ }^{40}$,

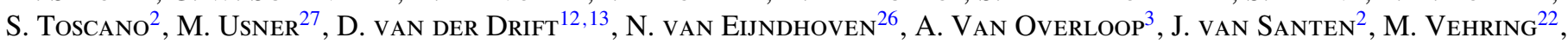
M. Voge ${ }^{27}$, M. Vraeghe ${ }^{3}$, C. Walck ${ }^{25}$, T. Waldenmaier ${ }^{7}$, M. WallrafF $^{22}$, M. Walter ${ }^{4}$, R. Wasserman ${ }^{19}$, Ch. Weaver ${ }^{2}$, C. WendT ${ }^{2}$, S. WesterhofF ${ }^{2}$, N. Whitehorn ${ }^{2}$, K. Wiebe ${ }^{11}$, C. H. Wiebusch ${ }^{22}$, D. R. Williams ${ }^{40}$, H. Wissing ${ }^{20}$, M. Wolf $^{25}$,

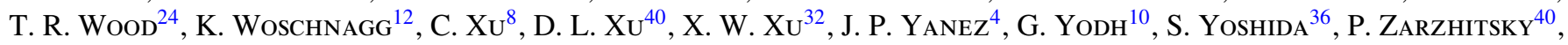
J. ZiemanN ${ }^{30}$, S. Zierke ${ }^{22}$, A. Zilles ${ }^{22}$, AND M. Zoll ${ }^{25}$

(ICeCube Collaboration)

${ }^{1}$ School of Chemistry \& Physics, University of Adelaide, Adelaide, SA 5005 Australia

${ }^{2}$ Department of Physics and Wisconsin IceCube Particle Astrophysics Center, University of Wisconsin, Madison, WI 53706, USA

${ }^{3}$ Department of Physics and Astronomy, University of Gent, B-9000 Gent, Belgium

${ }^{4}$ DESY, D-15735 Zeuthen, Germany

5 Department of Physics and Astronomy, University of Canterbury, Private Bag 4800, Christchurch, New Zealand

${ }^{6}$ Département de physique nucléaire et corpusculaire, Université de Genève, CH-1211 Genève, Switzerland

${ }^{7}$ Institut für Physik, Humboldt-Universität zu Berlin, D-12489 Berlin, Germany

${ }^{8}$ Bartol Research Institute and Department of Physics and Astronomy, University of Delaware, Newark, DE 19716, USA

${ }^{9}$ Physics Department, South Dakota School of Mines and Technology, Rapid City, SD 57701, USA

${ }^{10}$ Department of Physics and Astronomy, University of California, Irvine, CA 92697, USA

${ }^{11}$ Institute of Physics, University of Mainz, Staudinger Weg 7, D-55099 Mainz, Germany

${ }^{12}$ Department of Physics, University of California, Berkeley, CA 94720, USA

${ }^{13}$ Lawrence Berkeley National Laboratory, Berkeley, CA 94720, USA

${ }^{14}$ Department of Physics and Center for Cosmology and Astro-Particle Physics, Ohio State University, Columbus, OH 43210, USA

${ }^{15}$ Department of Astronomy, Ohio State University, Columbus, OH 43210, USA

${ }^{16}$ Science Faculty CP230, Université Libre de Bruxelles, B-1050 Brussels, Belgium

${ }^{17}$ Fakultät für Physik \& Astronomie, Ruhr-Universität Bochum, D-44780 Bochum, Germany

${ }^{18}$ Department of Physics, University of Wuppertal, D-42119 Wuppertal, Germany

${ }^{19}$ Department of Physics, Pennsylvania State University, University Park, PA 16802, USA

${ }^{20}$ Department of Physics, University of Maryland, College Park, MD 20742, USA

${ }^{21}$ Department of Physics and Astronomy, University of Kansas, Lawrence, KS 66045, USA

${ }^{22}$ III. Physikalisches Institut, RWTH Aachen University, D-52056 Aachen, Germany

${ }^{23}$ Department of Physics and Astronomy, Uppsala University, Box 516, S-75120 Uppsala, Sweden

${ }^{24}$ Department of Physics, University of Alberta, Edmonton, Alberta T6G 2G7, Canada 


\author{
${ }^{25}$ Oskar Klein Centre and Department of Physics, Stockholm University, SE-10691 Stockholm, Sweden \\ ${ }^{26}$ Vrije Universiteit Brussel, Dienst ELEM, B-1050 Brussels, Belgium \\ ${ }^{27}$ Physikalisches Institut, Universität Bonn, Nussallee 12, D-53115 Bonn, Germany \\ ${ }^{28}$ Laboratory for High Energy Physics, École Polytechnique Fédérale, CH-1015 Lausanne, Switzerland \\ ${ }^{29}$ School of Physics and Center for Relativistic Astrophysics, Georgia Institute of Technology, Atlanta, GA 30332, USA \\ ${ }^{30}$ Department of Physics, TU Dortmund University, D-44221 Dortmund, Germany \\ ${ }^{31}$ Department of Astronomy and Astrophysics, Pennsylvania State University, University Park, PA 16802, USA \\ ${ }^{32}$ Department of Physics, Southern University, Baton Rouge, LA 70813, USA \\ ${ }^{33}$ Department of Astronomy, University of Wisconsin, Madison, WI 53706, USA \\ ${ }^{34}$ T.U. Munich, D-85748 Garching, Germany \\ ${ }^{35}$ Los Alamos National Laboratory, Los Alamos, NM 87545, USA \\ ${ }^{36}$ Department of Physics, Chiba University, Chiba 263-8522, Japan \\ ${ }^{37}$ CTSPS, Clark-Atlanta University, Atlanta, GA 30314, USA \\ ${ }^{38}$ Department of Physics and Astronomy, Stony Brook University, Stony Brook, NY 11794-3800, USA \\ ${ }^{39}$ Université de Mons, 7000 Mons, Belgium \\ ${ }^{40}$ Department of Physics and Astronomy, University of Alabama, Tuscaloosa, AL 35487, USA \\ ${ }^{41}$ Department of Physics, University of Wisconsin, River Falls, WI 54022, USA \\ ${ }^{42}$ Sezione INFN, Dipartimento di Fisica, I-70126, Bari, Italy \\ ${ }^{43}$ Department of Physics and Astronomy, University of Alaska Anchorage, 3211 Providence Dr., Anchorage, AK 99508, USA \\ ${ }^{44}$ Department of Physics, University of Oxford, 1 Keble Road, Oxford OX1 3NP, UK \\ ${ }^{45}$ NASA Goddard Space Flight Center, Greenbelt, MD 20771, USA \\ Received 2012 October 18; accepted 2012 December 16; published 2013 February 15
}

\begin{abstract}
We report on the observation of anisotropy in the arrival direction distribution of cosmic rays at $\mathrm{PeV}$ energies. The analysis is based on data taken between 2009 and 2012 with the IceTop air shower array at the south pole. IceTop, an integral part of the IceCube detector, is sensitive to cosmic rays between $100 \mathrm{TeV}$ and $1 \mathrm{EeV}$. With the current size of the IceTop data set, searches for anisotropy at the $10^{-3}$ level can, for the first time, be extended to $\mathrm{PeV}$ energies. We divide the data set into two parts with median energies of $400 \mathrm{TeV}$ and $2 \mathrm{PeV}$, respectively. In the low energy band, we observe a strong deficit with an angular size of about $30^{\circ}$ and an amplitude of $\left(-1.58 \pm 0.46_{\text {stat }} \pm 0.52_{\text {sys }}\right) \times 10^{-3}$ at a location consistent with previous observations of cosmic rays with the IceCube neutrino detector. The study of the high energy band shows that the anisotropy persists to PeV energies and increases in amplitude to $\left(-3.11 \pm 0.38_{\text {stat }} \pm 0.96_{\text {sys }}\right) \times 10^{-3}$.
\end{abstract}

Key words: astroparticle physics - cosmic rays

Online-only material: color figures, figure set

\section{INTRODUCTION AND MOTIVATION}

Over the last half-decade, several experiments in the northern and southern hemispheres have reported anisotropy in the arrival direction distribution of cosmic rays at $\mathrm{TeV}$ energies. In the northern sky, two features dominate the $\mathrm{TeV}$ cosmic-ray sky: a large-scale structure with an amplitude of about $10^{-3}$ usually described as a dipole (Munakata et al. 1997; Amenomori et al. 2005, 2006; Guillian et al. 2007; Abdo et al. 2009) and a smallscale structure with a few hot spots of angular size $10^{\circ}$ to $30^{\circ}$ (Abdo et al. 2008; Vernetto et al. 2009).

In the southern hemisphere, cosmic-ray arrival directions at $\mathrm{TeV}$ energies observed with the IceCube neutrino detector at the south pole exhibit features similar to those discovered in the northern sky. In a data set with a median energy of $20 \mathrm{TeV}$, the arrival direction distribution exhibits a large-scale structure similar in orientation and shape to the large-scale feature observed in the northern sky (Abbasi et al. 2010b).

In addition, there is a small-scale structure which is about a factor of five weaker in relative intensity than the large-scale structure. This small-scale structure contains several regions of significant cosmic-ray excess and deficit (Abbasi et al. 2011).

There are several models that can at least qualitatively explain the anisotropy. Cosmic rays in this energy range are assumed to be accelerated in Galactic sources, most likely in shocks from supernova explosions. The transport of cosmic rays at these energies in the Galactic magnetic field is diffusive, and the flux from a single nearby source would be observed on Earth as a dipole with its maximum possibly oriented toward the source.
If a few supernova remnants from recent (10-100 kyr) nearby supernovae were primarily responsible for the Galactic cosmicray flux (Erlykin \& Wolfendale 2006), their combined flux on Earth would be a superposition of these individual dipoles. The observed large-scale structure in the cosmic-ray flux could be the sum of the contributions from a few nearby sources and from the large-scale distribution of supernova remnants in our Galaxy (Blasi \& Amato 2012; Pohl \& Eichler 2012). Our limited knowledge of nearby supernova remnants renders a more quantitative explanation of the amplitude and the phase of the observed large-scale anisotropy impossible, but among the predictions of this model is an increase of the anisotropy with the energy of the primary cosmic rays.

The small-scale structure is more difficult to explain. The Larmor radius of a proton with $10 \mathrm{TeV}$ energy in a magnetic field with $\mu \mathrm{G}$ strength (an estimate of the strength of magnetic fields in our Galaxy; Han et al. 2006) is only of order $0.01 \mathrm{pc}$, so the observed small-scale anisotropy cannot correlate with nearby sources. Recent studies link the smaller structure to cosmic-ray propagation in turbulent magnetic fields within a few tens of parsecs from Earth (Giacinti \& Sigl 2012). In this case, the small-scale structure is also expected to show a dependence on energy.

Several experiments have studied the energy dependence of the anisotropy. In the northern hemisphere, the EAS-TOP experiment reports anisotropy up to at least $400 \mathrm{TeV}$. The data contain weak evidence for an increase in the amplitude of the anisotropy as a function of energy, as well as a change of phase (Aglietta et al. 2009). Measurements in the southern hemisphere 
with IceCube also indicate that anisotropy is still present in a data set with $400 \mathrm{TeV}$ median energy. It differs in shape and strength from the anisotropy observed at $20 \mathrm{TeV}$ and is no longer a superposition of large- and small-scale structures, but rather dominated by a single deficit region with an angular size of about $30^{\circ}$ (Abbasi et al. 2012).

IceCube is primarily a neutrino detector designed to search for sources of astrophysical neutrinos. The data set used in the cosmic-ray analysis consists of downgoing atmospheric muons from cosmic-ray air showers in the atmosphere above the IceCube detector. The downgoing muons preserve the direction of the cosmic-ray primary, but the muon energy is only a poor indicator of the air shower energy. In addition, because of the high muon rate $\left(10^{6}\right.$ times the neutrino rate in IceCube), these events are stored in a separate data format which only contains the results of a fast online reconstruction performed at the south pole. No raw data are preserved. An offline reconstruction with more sophisticated algorithms to determine the muon energy is therefore not possible.

Above $1 \mathrm{TeV}$, the energy resolution is of order 0.5 in $\log (E)$ (Abbasi et al. 2012). The energy resolution is estimated as the standard deviation of the distribution of the difference $\log \left(E_{\text {true }}\right)-\log \left(E_{\text {reco }}\right)$, where $E_{\text {true }}$ and $E_{\text {reco }}$ are the true and reconstructed shower energies obtained from simulation studies, respectively. This distribution has substantial tails, making it difficult to isolate a set of events with large median energy that is not contaminated by low-energy events.

The IceTop air shower array is located at $2835 \mathrm{~m}$ altitude on the surface of the ice sheet above the IceCube neutrino detector. IceTop is a dedicated cosmic-ray detector optimized for air shower observations at $\mathrm{PeV}$ energies. IceTop is used to record not only the muonic component of the air showers, but also the electromagnetic component, at ground level. With the sparse sampling of the shower front typical for air shower arrays, it also has a considerably higher detection threshold for cosmic rays than IceCube. The size and geometry of the array result in a threshold for reconstruction of air showers of approximately $300 \mathrm{TeV}$.

As an air shower array, IceTop provides a more measured information per shower than IceCube. A study of cosmic-ray anisotropy with IceTop can therefore complement measurements with IceCube. With its high energy threshold, an energy resolution better than 0.1 in $\log (E)$ (Abbasi et al. 2013), and sensitivity to the cosmic-ray composition, IceTop data are particularly useful for studying anisotropy at energies above $1 \mathrm{PeV}$. Due to the lower data rate, the full event information is stored, and a more careful offline reconstruction of the primary cosmicray properties is possible.

The accumulated IceTop data set is not yet large enough for a detailed study of per-mille anisotropy in several energy bins. However, the statistics collected between 2009 and 2012 are now sufficient to search for anisotropy in two energy bands centered at $400 \mathrm{TeV}$ and $2 \mathrm{PeV}$. The $400 \mathrm{TeV}$ data set can be compared to results based on downgoing muons in IceCube at a similar energy but for a different cosmic-ray composition model (Abbasi et al. 2012). With the $2 \mathrm{PeV}$ data set, the search for anisotropy is extended to energies not previously explored. In this paper, we report on the observation of anisotropy with IceTop at both energies.

The paper is organized as follows. In Section 2, we describe the IceTop detector and the data sample used in this analysis. The analysis techniques and the results are briefly described in Section 3. The analysis is based on methods used in previous

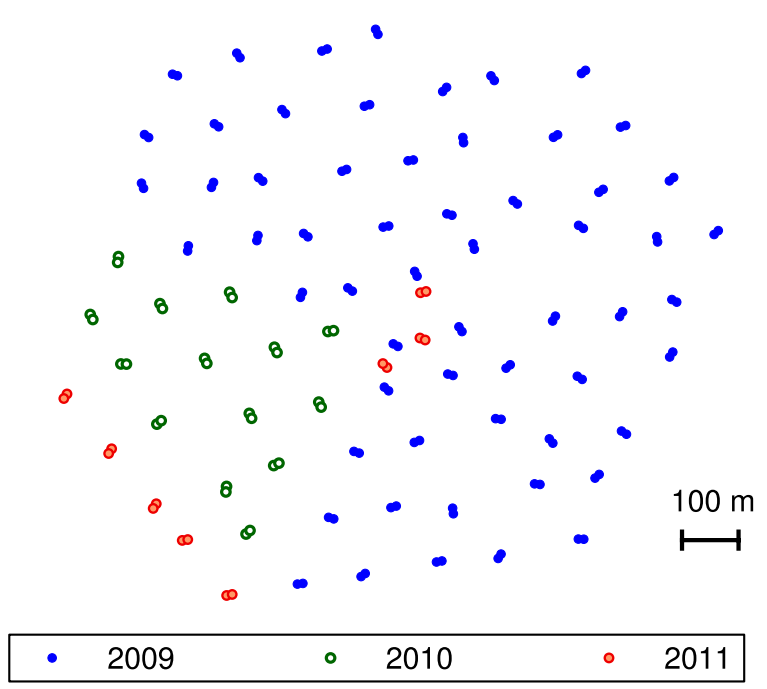

Figure 1. Detector configurations of the IceTop array, 2009-2011. IT59 comprised 59 stations deployed through 2009 January (blue circles). In 2009 and 2010, 14 additional stations were deployed and the detector was operated in the IT73 configuration (blue and green circles). The remaining eight stations (red circles) were deployed in late 2010. The final IceTop configuration (IT81) consists of 81 stations and operated in 2011.

(A color version of this figure is available in the online journal.)

work; a more detailed description of these techniques can be found in Abbasi et al. (2011). Section 4 discusses systematic uncertainties, and Section 5 summarizes the paper.

\section{DETECTOR, DATA SETS, AND SIMULATION}

\subsection{The IceTop Detector}

The IceTop cosmic-ray air shower array consists of 81 stations distributed over an area of $1 \mathrm{~km}^{2}$ in a hexagonal grid with a distance of about $125 \mathrm{~m}$ between neighboring stations. During the construction phase of IceTop between 2005 and 2010, the detector was operated in several partial configurations. In this work we use data taken during three periods: between 2009 May and 2010 May when the detector was operated in a 59-station configuration (IT59); between 2010 May and 2011 May when IceTop operated with 73 stations (IT73); and between 2011 May and 2012 May when the detector operated in its final 81station configuration (IT81). The layout of these configurations is shown in Figure 1.

IceTop is described in detail in Abbasi et al. (2013). Each IceTop station is instrumented with two light-tight ice Cherenkov tanks separated by about $10 \mathrm{~m}$. Each tank is $1.8 \mathrm{~m}$ in diameter, $1.3 \mathrm{~m}$ in height, and is filled with transparent ice up to a height of $0.9 \mathrm{~m}$. Frozen into the ice are two Digital Optical Modules (DOMs) that are used to detect the Cherenkov radiation emitted by charged leptons present in the cosmic-ray air shower. A DOM consists of a glass sphere that houses a 10" Hamamatsu photomultiplier tube (PMT; Abbasi et al. 2010a), together with electronic boards used for filtering, digitization, and readout (Abbasi et al. 2009).

The two DOMs inside each IceTop tank are operated at different PMT gains in order to increase the dynamic range of the detector. The high-gain DOMs in the two tanks that form a station are run in local coincidence mode, and data readout is enabled if one of the DOMs records photon hits within $\pm 1 \mu \mathrm{s}$ of the other. The trigger condition in IceTop requires at least six DOMs to have recorded locally coincident hits within a 


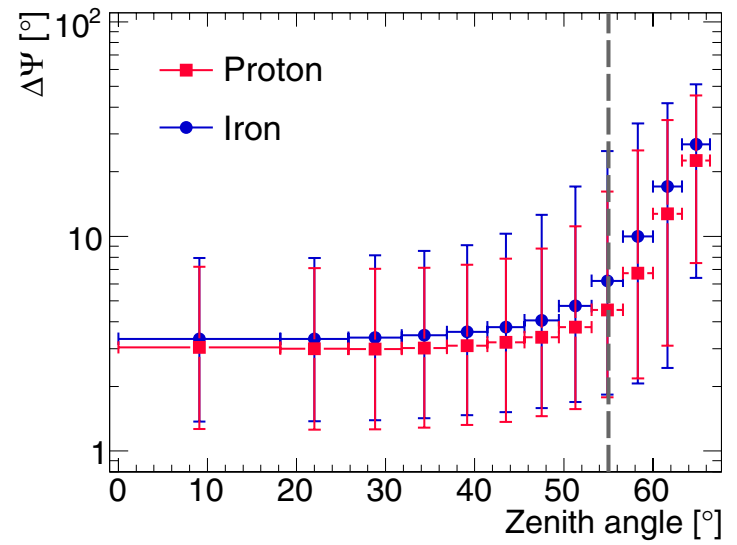

Figure 2. Median opening angle $\Delta \Psi$ between reconstructed and true arrival direction as a function of reconstructed zenith angle $\theta$. At large zenith angles the fraction of misreconstructed events increases. For this reason, a zenith cut was implemented that restricts the analysis to events with $\theta<55^{\circ}$ (dashed gray line). The error bars correspond to a $68 \%$ containing interval. IT59, IT73, and IT81 show the same dependence of angular resolution on reconstructed zenith angle.

(A color version of this figure is available in the online journal.)

time window of $5 \mu \mathrm{s}$, which implies that at least two stations participated in the event.

Due to the limited bandwidth available for data transmission from the south pole, events triggering less than eight stations were prescaled by a factor of eight during the operation of IT59, and by a factor of three during IT73 and IT81. Events triggering more than eight stations were not prescaled.

\subsection{Data Sample and Simulation}

The prescaling scheme described above was used to divide the data into two samples: a "low-energy" data set, containing events with at least three but less than eight stations triggered, and a "high-energy" data set that contains events where eight or more stations were triggered.

During the operation of IT59, IT73, and the first year of IT81, a total of $3.55 \times 10^{8}$ events with more than three triggered stations were recorded. Of these events, $2.90 \times 10^{8}$ were classified as "low-energy" events, while the "high-energy" sample contains the remaining $0.65 \times 10^{8}$ events. A zenith angle cut (described below) was used to remove misreconstructed events at large zenith angles. This cut reduced the final sample to $2.86 \times 10^{8}$ events in the low-energy set and $0.64 \times 10^{8}$ events in the high-energy sample.

The angular resolution of the shower reconstruction algorithm and the median energy of the data sets were determined using simulated cosmic-ray air showers. Events were generated with the CORSIKA Monte Carlo code (Heck et al. 1998) and passed through a full simulation of the IceTop detector (Abbasi et al. 2013). The median energy of the samples determined using this simulation will depend on the assumptions made about the chemical composition of the primary cosmic rays. The detailed primary composition has not been directly measured for energies beyond $100 \mathrm{TeV}$, but models that extrapolate existing measurements to higher energies indicate that in the energy range of this analysis, the cosmic-ray flux consists mainly of protons, helium, and iron (Gaisser 2012). Their relative contribution is a function of energy, with helium and protons dominating around $100 \mathrm{TeV}$ and iron becoming the dominant element above several tens of PeV. Given the uncertainties in the composition, we have generated only proton and iron showers

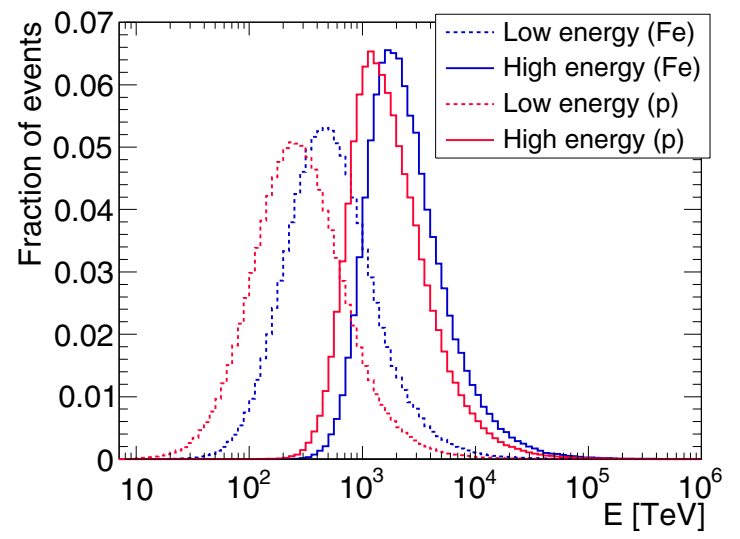

Figure 3. Simulated energy distributions for all events in the low-energy (dashed) and high-energy (solid) data sets assuming all-iron (blue) and allproton (red) compositions. The energy distributions are the same for the IT59, IT73, and IT81 configurations.

(A color version of this figure is available in the online journal.)

Table 1

Median Energy and 68\% Containing Interval in PeV for the Two Energy Bands Used in This Work Assuming That the Cosmic Rays Consists of Either Protons or Iron Nuclei

\begin{tabular}{lccccc}
\hline \hline \multirow{2}{*}{ Composition } & \multicolumn{2}{c}{ Low Energy } & & \multicolumn{2}{c}{ High Energy } \\
\cline { 2 - 3 } \cline { 6 - 7 } & $\tilde{E}$ & $68 \%$ Interval & & $\tilde{E}$ & $68 \%$ Interval \\
\hline Proton & $0.27 \mathrm{PeV}$ & $0.11-0.69 \mathrm{PeV}$ & & $1.6 \mathrm{PeV}$ & $0.83-3.8 \mathrm{PeV}$ \\
Iron & $0.50 \mathrm{PeV}$ & $0.22-1.2 \mathrm{PeV}$ & & $2.2 \mathrm{PeV}$ & $1.2-5.3 \mathrm{PeV}$ \\
\hline
\end{tabular}

as the two limiting cases for the chemical composition. The true median energy of the sample should be contained in the interval defined by these two cases.

The arrival direction of cosmic-ray showers in IceTop is reconstructed by fitting a plane to the front of the air shower. The fit algorithm implements an analytic $\chi^{2}$-minimization that uses the positions and hit times of the triggered stations to reconstruct the direction vector of the shower. From simulation we have determined the median angular resolution of this algorithm to be $3^{\circ}$ for both proton and iron showers for all detector configurations. In other IceTop analyses, a more precise reconstruction algorithm is used that takes into account the curvature of the air shower front and can reach a sub-degree resolution. The plane fit is better suited to our needs since it provides a resolution that is several times smaller than the typical angular scale of the anisotropic pattern $\left(>20^{\circ}\right)$ without requiring a larger number of stations triggered which would reduce the size of the cosmic-ray sample. As shown in Figure 2, the resolution of the plane fit degrades rapidly for showers with zenith angles larger than $60^{\circ}$. For this reason, this analysis is limited to events with a reconstructed zenith angle smaller than $55^{\circ}$.

The median energies of the data sets were determined from the energy distribution of the simulated air showers which satisfy the same trigger conditions as events in the low- and high-energy data samples. The simulated energy distributions are shown in Figure 3 . The median energies and the $68 \%$ containing intervals for the two composition models for each energy band are shown in Table 1, which shows that the low energy band has a median energy in the range $270-500 \mathrm{TeV}$, while the median energy for the high-energy sample should be contained in the range 1.6-2.2 PeV. 

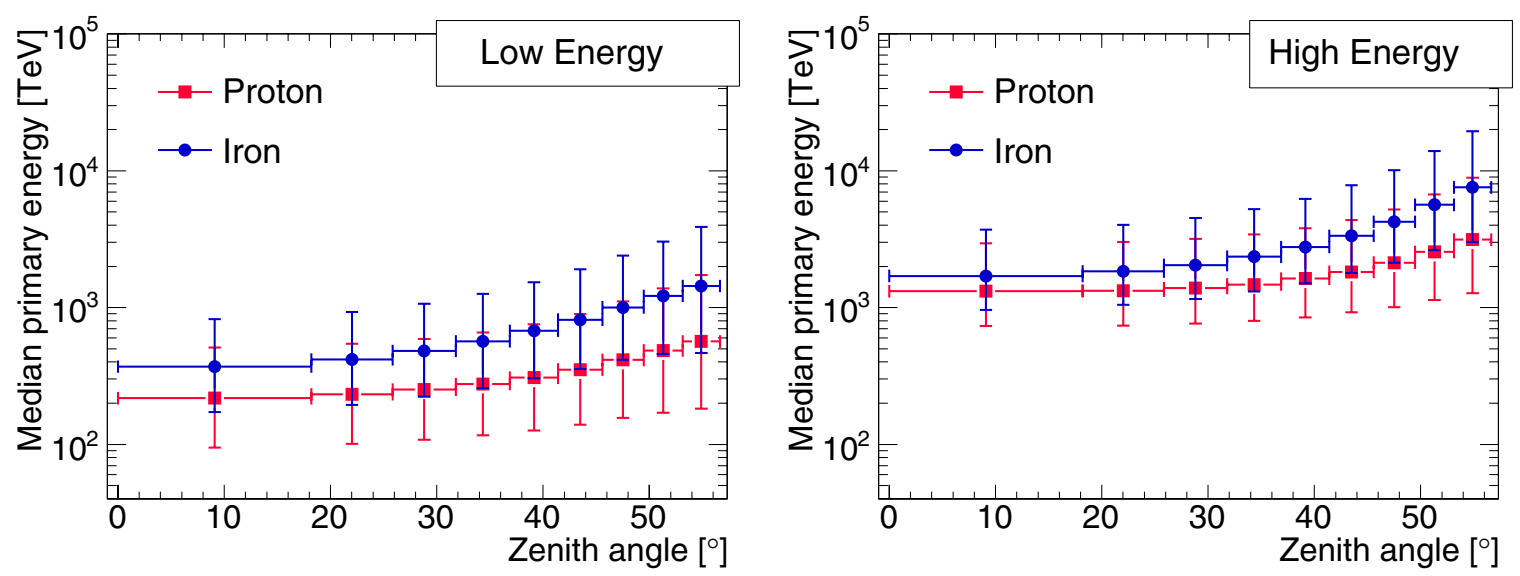

Figure 4. Median energy as a function of reconstructed zenith angle for the low-energy (left), and high-energy (right) data sets for proton and iron cosmic-ray primaries. The error bars correspond to a $68 \%$ containing interval. IT59, IT73, and IT81 show the same energy dependence on the reconstructed zenith angle.

(A color version of this figure is available in the online journal.)

For both data samples, the median energy of the primary cosmic rays monotonically increases with zenith angle, as illustrated in Figure 4.

\section{DATA ANALYSIS AND RESULTS}

\subsection{Map Making Procedure}

The search for anisotropy in the IceTop data is based on techniques applied in the analysis of cosmic-ray data with IceCube and described in more detail in Abbasi et al. (2011). To obtain a skymap of the relative intensity of the cosmic-ray flux, the map of reconstructed arrival directions in equatorial coordinates is compared to an estimate of the isotropic expectation represented by a "reference map." The reference map is constructed from the data using the time-scrambling method (Alexandreas et al. 1993). For each detected event, 20 "fake" events are generated by keeping the local zenith and azimuth angles fixed and calculating new values for equatorial coordinates using event times randomly selected from within a time window $\Delta t$ bracketing the event. The fake events are stored in the reference map with a weight of $1 / 20$. In order to be sensitive to anisotropy at all angular scales, we use $\Delta t=24 \mathrm{hr}$. The stability of the detector over this time was verified by performing a $\chi^{2}$ test where the distributions of zenith and azimuth coordinates for the events are compared inside the window.

By using the time-scrambling algorithm, the events included in the reference map have the same arrival direction distribution as the data in local coordinates. In addition, the method compensates for variations in the event rate, including gaps in the detector uptime. We note, however, that the method is known to create artificial deficits near strong excesses, and vice versa near large deficits. With a $24 \mathrm{hr}$ scrambling window, a single strong excess (deficit) will raise (lower) the reference map level for the entire right ascension band at the declination of the excess (deficit) which could bias the observed amplitude of the anisotropy and its statistical significance. This effect can become important if extended regions of strong excess or deficit flux are present in the data, and the resulting skymaps should be interpreted carefully with this potential bias in mind.

The maps are built using the HEALPix equal-area pixelization of the sphere (Gorski et al. 2005) with an average pixel size of about 0.9. Maps of statistical significance for the low- and high-energy data sets are shown in Figure 5. The bin size is not optimized for a study of anisotropy at scales larger than
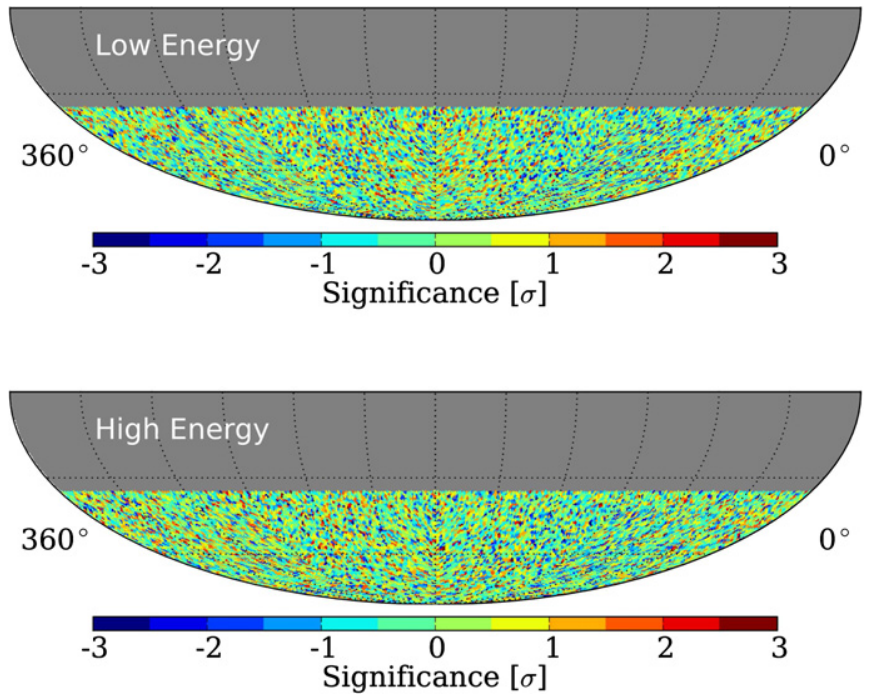

Figure 5. Maps of statistical significance for the low-energy (top) and highenergy (bottom) data sets with no smoothing applied.

(A color version of this figure is available in the online journal.)

the angular resolution of the detector. We therefore apply a smoothing procedure to both the data map and the reference map in order to increase the sensitivity of the method to structures with larger angular sizes. The smoothing procedure is essentially a rebinning of the maps, but rather than producing maps with fewer (but statistically independent) pixels, we retain the original 0.9 binning. At each bin, we add the counts from all pixels within some angular radius ("smoothing radius") of the bin. This produces maps with Poisson uncertainties, but with bins that are not statistically independent. Since the optimal smoothing scale is not known a priori, we perform a scan over different smoothing angles. After smoothing, the relative intensity $\delta I_{i}$, i.e., the amplitude of deviations from the isotropic expectation for each angular bin $i$, is calculated using

$$
\delta I_{i}=\frac{\Delta N_{i}}{\langle N\rangle_{i}}=\frac{N_{i}-\langle N\rangle_{i}}{\langle N\rangle_{i}},
$$

where $N_{i}$ and $\langle N\rangle_{i}$ are the number of events in pixel $i$ of the data map and the reference map, respectively. The statistical significance of the observed deviations is calculated using the method described in Li \& Ma (1983). 

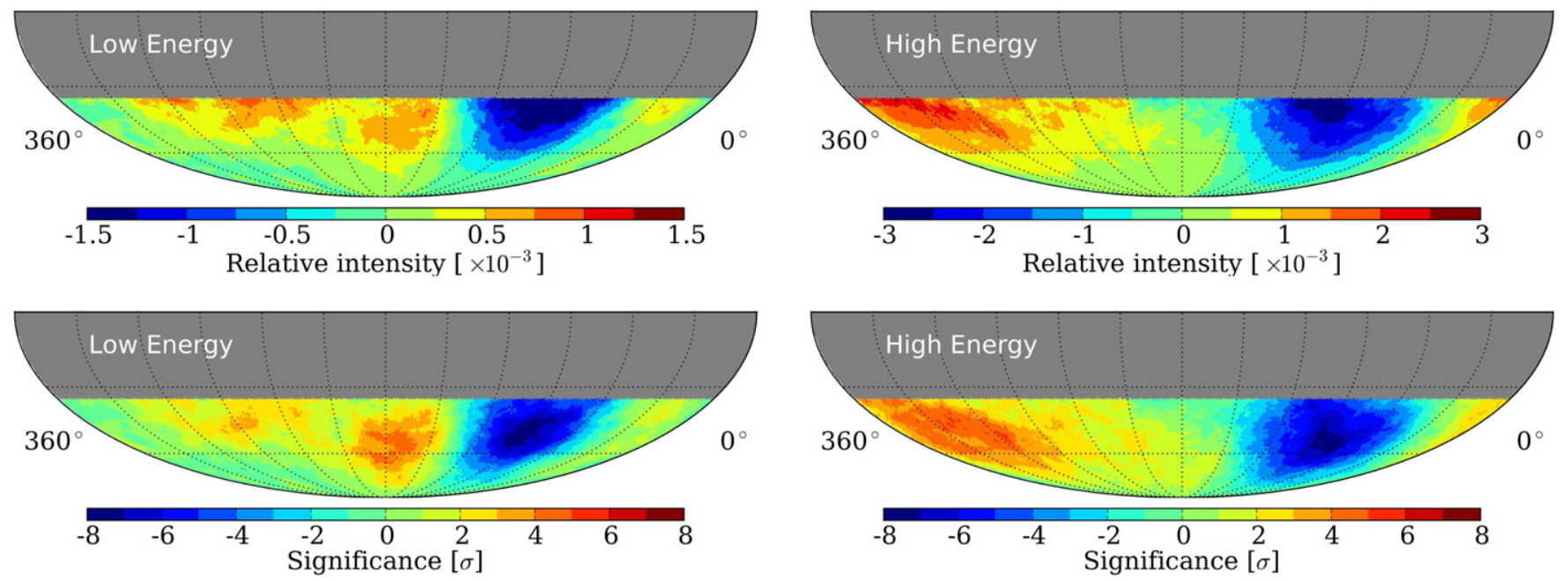

Figure 6. Relative intensity (top) and statistical significance (bottom) maps for the low-energy (left) and high-energy (right) data sets for a smoothing angle of $20^{\circ}$. (The complete figure set (64 images) and a color version of this figure are available in the online journal.)

The significance is later corrected to account for the number of trials introduced by looking everywhere in the sky for significant fluctuations, and for the fact that a scan was performed over different smoothing radii to search for anisotropy at different angular scales.

\subsection{Results}

The smoothing procedure described above was applied to the low- and high-energy maps for smoothing radii between $5^{\circ}$ and $50^{\circ}$ in $3^{\circ}$ steps. A search for regions of high significance was performed on the resulting smoothed maps. The relative intensity and significance maps for the low- and high-energy data are shown in Figure 6 for a representative smoothing radius of $20^{\circ}$ where all the relevant features observed in these two energy ranges are visible. Maps for all other smoothing radii are given in Figure 6.

The low-energy map is dominated by a strong deficit in relative intensity. The statistical significance of the deficit reaches a maximum of $8.5 \sigma$ for a smoothing radius of $29^{\circ}$ at a location around $(\alpha=85.8, \delta=-36.4)$. Since the search for this minimum is performed over about 10,000 pixels in the map, and across all 16 different smoothing radii, there is a trials factor of at most $1.6 \times 10^{5}$ that reduces the post-trial significance of the deficit to $7.0 \sigma$. It must be noted that this correction for trials is conservative, since the pixels in the map are statistically correlated by the smoothing procedure, which results in a smaller effective number of trials than the maximum.

For the optimal smoothing radius of $29^{\circ}$, the relative intensity $\delta I$ reaches a value of about $-1.5 \times 10^{-3}$ at the location of the greatest deficit around $(\alpha=83.7, \delta=-35.7)$, near the edge of our exposure window. Differences in declination between the location of the maximum relative intensity and maximum significance are due to the fact that the statistical significance accounts for both signal strength and the declination-dependence of our statistics. This usually implies that the position of maximum significance is offset toward lower declination values where the statistics increase.

Also visible in the low-energy map is a region of excess flux located around $(\alpha=182.9, \delta=-55.9)$. The maximum pre-trial significance for this region is $5.3 \sigma$ for a smoothing angle of $26^{\circ}$. The significance falls below the $3 \sigma$ threshold after accounting for trials.
As mentioned above, in the presence of a strong deficit the time-scrambling algorithm can introduce an underestimation of the isotropic reference level, which can produce spurious excess regions in the parts of the sky surrounding the signal region. For this reason, it is possible that the excess observed in the lowenergy data set is associated with the presence of the deficit region.

The high-energy map also shows statistically significant anisotropy which is dominated by a deficit located in the same approximate position as that observed in the low-energy data. The pre-trial significance of the deficit is $8.6 \sigma$ ( $7.1 \sigma$ post-trials) for a smoothing angle of $35^{\circ}$, with its minimum located at $(\alpha=79.4, \delta=-37.2)$. The main difference between the lowand high-energy deficits is that the value of $\delta I$ for the greatest deficit in the high-energy sample is $-2.3 \times 10^{-3}$, larger than its low-energy counterpart. This is evident in Figure 7, where the relative intensity is projected onto the right ascension axis using the declination band $-75^{\circ}<\delta<-35^{\circ}$.

A second notable feature in the high-energy map is a wide excess region that reaches a peak significance of $5.9 \sigma(3.4 \sigma$ post-trials) for a smoothing angle of $41^{\circ}$. The excess does not appear to be concentrated in any particular part of the sky, but distributed across a wide band in right ascension. This is visible in the one-dimensional projection shown in Figure 7, where the relative intensity reaches a plateau above $\alpha>170^{\circ}$ which is offset from zero by about $10^{-3}$. As in the low-energy data set, such an excess could be associated with the presence of the observed deficit in the same declination band that introduces a bias in the reference-level estimation.

In order to characterize the observed anisotropic pattern, we attempted to fit the relative intensity projections of the data shown in Figure 7 with the first terms (dipole and quadrupole) of a harmonic series:

$$
\delta I(\alpha)=\sum_{j=1}^{2} A_{j} \cos \left[j\left(\alpha-\phi_{j}\right)\right]+B .
$$

The harmonic fit parameters for the low-energy data set are $A_{1}=(5.53 \pm 0.91) \times 10^{-4}, \phi_{1}=-111.7 \pm 9.7, A_{2}=(4.03 \pm$ $0.80) \times 10^{-4}, \phi_{2}=1.0 \pm 7.9$, and $B=(-0.47 \pm 0.66) \times 10^{-4}$. For all parameters the indicated uncertainties are statistical. The $\chi^{2} /$ dof for the fit is $18.4 / 10$. 

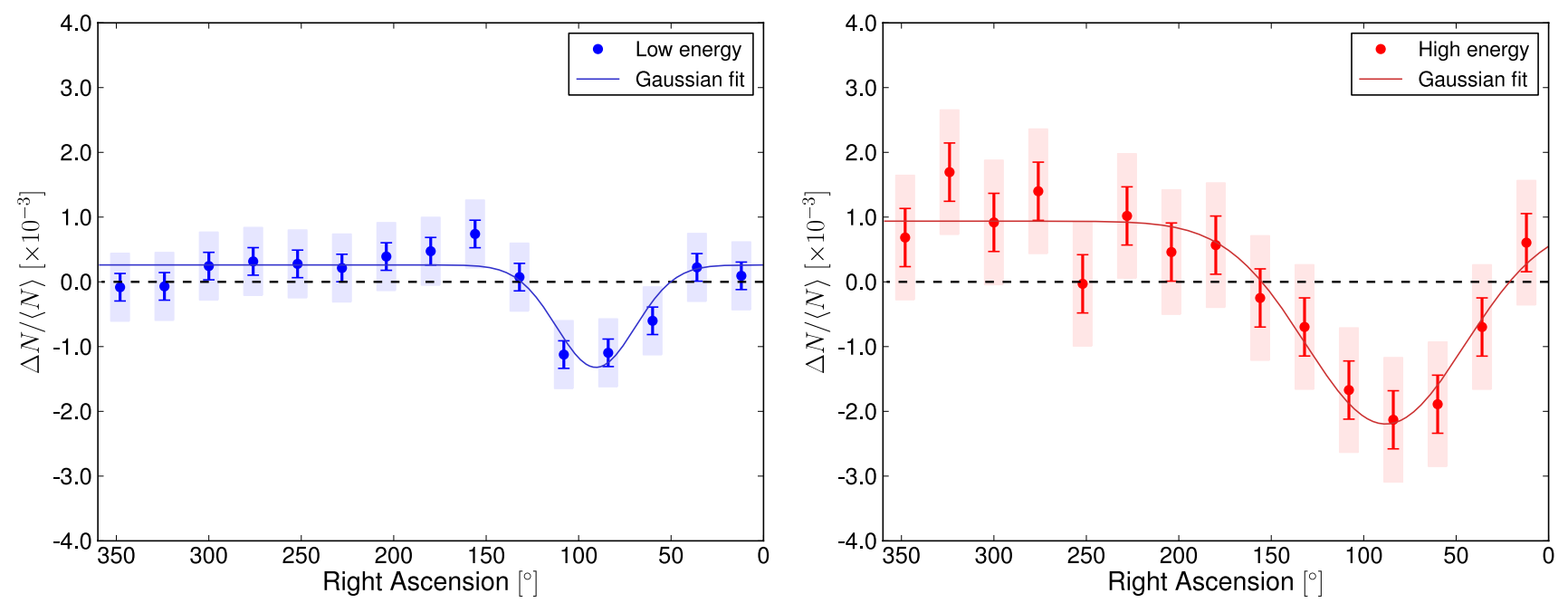

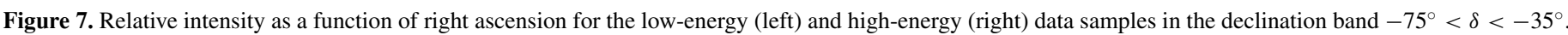

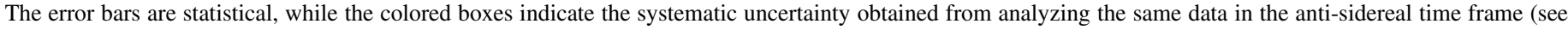
Section 4 for details). The result of a fit using the Gaussian function given in Equation (3) to both energy bands is also shown.

(A color version of this figure is available in the online journal.)

Table 2

Fit Parameters Obtained for Both Energy Data sets for the Gaussian Function Given in Equation (3)

\begin{tabular}{lcc}
\hline \hline & Low Energy & High Energy \\
\hline$A$ & $(-1.58 \pm 0.46 \pm 0.52) \times 10^{-3}$ & $(-3.11 \pm 0.38 \pm 0.96) \times 10^{-3}$ \\
$\alpha_{s}$ & $90.6 \pm 6.8 \pm 9.3$ & $88.1 \pm 6.8 \pm 11.1$ \\
$\sigma$ & $21.3 \pm 5.8 \pm 7.6$ & $43.1 \pm 7.3 \pm 13.1$ \\
$b$ & $(2.61 \pm 0.64 \pm 5.20) \times 10^{-4}$ & $(9.37 \pm 1.96 \pm 9.60) \times 10^{-4}$ \\
$\chi^{2} /$ dof & $13.2 / 11$ & $10.7 / 11$ \\
\hline
\end{tabular}

Note. In all cases, the first quoted uncertainty is statistical, while the second one corresponds to the systematics.

In the case of the high-energy data set the fit parameters are $A_{1}=(1.43 \pm 0.19) \times 10^{-3}, \phi_{1}=-84.7 \pm 8.0$, $A_{2}=(7.34 \pm 1.9) \times 10^{-4}, \phi_{2}=-14.7 \pm 7.7$, and $B=$ $(-0.14 \pm 1.35) \times 10^{-4}$. In this case, the $\chi^{2} /$ dof for the fit is $6.3 / 10$.

In the case of the low-energy data set, the reduced $\chi^{2}$ of the fit is considerably larger than unity, indicating that for these data, as in the case of the previous observation of anisotropy at $400 \mathrm{TeV}$ with IceCube (Abbasi et al. 2012), this choice of harmonic base functions does not fit the data particularly well. For this reason, a new fit is performed using the following Gaussian function:

$$
\delta I(\alpha)=A e^{-\left(\frac{\alpha-\alpha_{s}}{\sqrt{2} \sigma}\right)^{2}}+b,
$$

where $\alpha$ is right ascension, $A$ is the amplitude, $\sigma$ is the width, and $\alpha_{s}$ is the right ascension of the center of the deficit. The parameter $b$ represents an overall offset from isotropy that can be introduced by the presence of a strong signal in the data.

The results of these fits are shown in Table 2, and indicate that while the center point of the deficit for both data sets is consistently located at $\alpha_{s} \sim 90^{\circ}$, both the amplitude and the width are larger in the high-energy sample, with both values increasing by about a factor of two with respect to the lowenergy case. The location of the deficit in the right ascension projection (Figure 7) is consistent with its location in the skymap (Figure 6), within statistical and systematic uncertainties, for both the low-energy and high-energy samples. Similarly, the amplitudes in relative intensity of the deficit agree well, when the overall offset $b$ is taken into account.

The systematic uncertainty associated with each fit value was obtained from the systematic uncertainty of the relative intensity projection, shown as shaded boxes in Figure 7. The systematic uncertainty of the projection was conservatively estimated as the maximum amplitude of the relative intensity distribution for each energy data set when analyzed in the anti-sidereal time frame (see Section 4).

A previous search for anisotropy as a function of cosmicray primary energy was performed using muon data from the IceCube detector (Abbasi et al. 2012). In that analysis, cuts were applied to the data to select two sets of cosmic-ray events: one with a median primary energy of $20 \mathrm{TeV}$ and a second one with a median energy of $400 \mathrm{TeV}$, similar to the lowenergy IceTop sample. The $400 \mathrm{TeV}$ IceCube skymap shows a deficit region similar to the one observed in the IceTop low-energy sample. At $20 \mathrm{TeV}$, IceCube observed a largescale structure that is consistent with previous observations at these energies (Abbasi et al. 2010b, 2011). The angular size and the orientation in the sky of the $20 \mathrm{TeV}$ anisotropy are different from that observed at $400 \mathrm{TeV}$ by IceCube and IceTop. Note that while the IceTop median energy was obtained by assuming two limiting cases of primary chemical composition (all-proton or all-iron primaries only), the IceCube median energy was obtained assuming that the cosmic-ray flux follows the polygonato composition models (Hörandel 2003), which in principle could lead to some differences in the actual median energy and the energy distribution of events in both samples.

The smoothing procedure described here was also used in the IceCube analysis, and the significance of the deficit was maximized for a smoothing radius of $29^{\circ}$, the same as the optimal smoothing angle for the low-energy IceTop data. Figure 8 shows a comparison between the IceCube and IceTop results at $400 \mathrm{TeV}$. The location and amplitude of the deficits observed in both data sets agree given the current values of the statistical and systematic uncertainties associated with both measurements. 


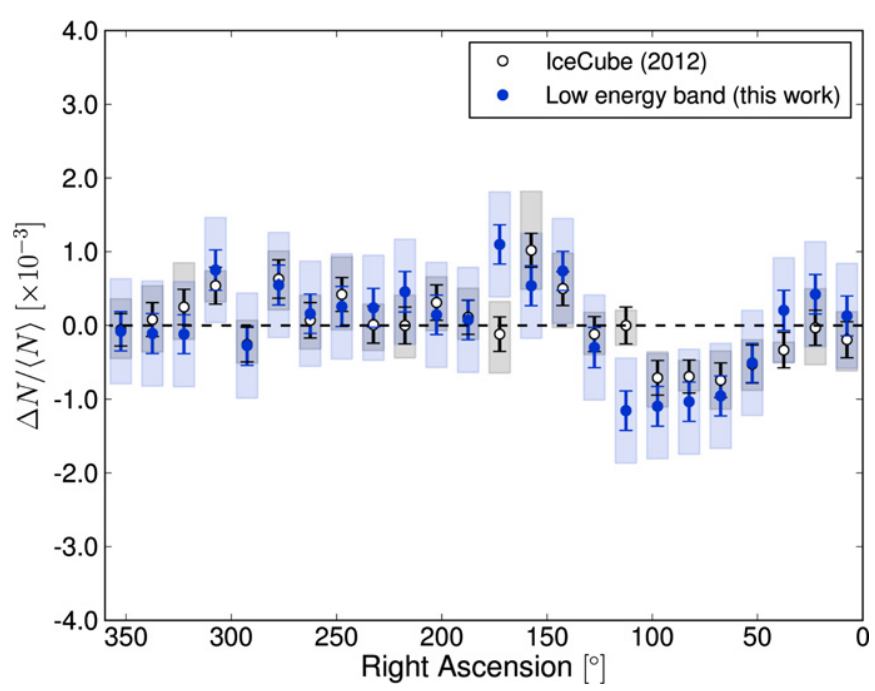

Figure 8. Comparison between the relative intensity projections for the IceTop low-energy sample (blue filled circles) and the IceCube $400 \mathrm{TeV}$ sample (black open circles) reported by Abbasi et al. (2012). The location and amplitude of both deficits are consistent given the statistical and systematic uncertainties. The declination range for the IceCube plot is $-75^{\circ}<\delta<-25^{\circ}$, slightly different from the IceTop one.

(A color version of this figure is available in the online journal.)

\section{SYSTEMATIC UNCERTAINTIES}

A number of tests have been performed in order to quantify the systematic uncertainties associated with the observation of anisotropy in the IceTop data.

In the first study, the anisotropy search was performed on three independent data subsamples, each containing events recorded during the operation of the three different detector configurations IT59, IT73, and IT81 considered in this work. In this manner we can determine the possible systematic effect introduced by the changing geometry of the detector on the observed anisotropy.

The results of this comparison are shown in Figure 9, where the relative intensity as a function of right ascension for the declination band $-75^{\circ}<\delta<-35^{\circ}$ is displayed for all three detector configurations and for the low- and high- energy samples separately. The anisotropy observed by all three configurations is consistent within statistical uncertainties.

Another test was performed to evaluate the impact of the seasonal variation of the cosmic-ray rate at the south pole (Tilav et al. 2009). In this study, four different time periods were selected from the data: June through August, September through November, December through February, and March through May for each year of operation of the detector. These four data sets contain events taken with comparable detector geometries, but recorded during different phases of the seasonal variation cycle. The results of this study indicate that the anisotropy observed in each of the four time periods is consistent within statistical uncertainties.

Other possible seasonal effects on the anisotropy are also investigated. First, an analysis was performed to look for anisotropy in the so-called solar time frame, defined as having 365.25 (i.e., complete revolutions in the coordinate frame) per year. The motion of the Earth around the Sun should create a dipolar anisotropy in the solar frame with an amplitude of $4.7 \times 10^{-4}$. No anisotropy was observed using IceTop data. However, simulations of the solar dipole assuming the IceTop acceptance in local coordinates indicate that the current size of the data set is insufficient for a statistically significant observation.

The second analysis consists of a search for anisotropy analysis in the "anti-sidereal" time frame, defined as having 364.25 days. No signal should be observed in this frame unless there exists a seasonal variation in the solar time frame that could affect the anisotropy in sidereal time (period of 366.25 days). See Abbasi et al. (2011) for details.

We performed the anti-sidereal analysis on the combined three-year data set and obtained both skymaps and onedimensional relative intensity projections for the low- and high energy bands. The skymaps produced for the anti-sidereal frame do not exhibit any significant anisotropy that could indicate a possible systematic bias in the sidereal frame. The systematic uncertainty of the sidereal anisotropy due to seasonal variations, shown in Figure 7, is obtained from the relative intensity projections in the anti-sidereal frame. This uncertainty is conservatively estimated as the maximum departure from the reference level of the anti-sidereal right ascension distribution.
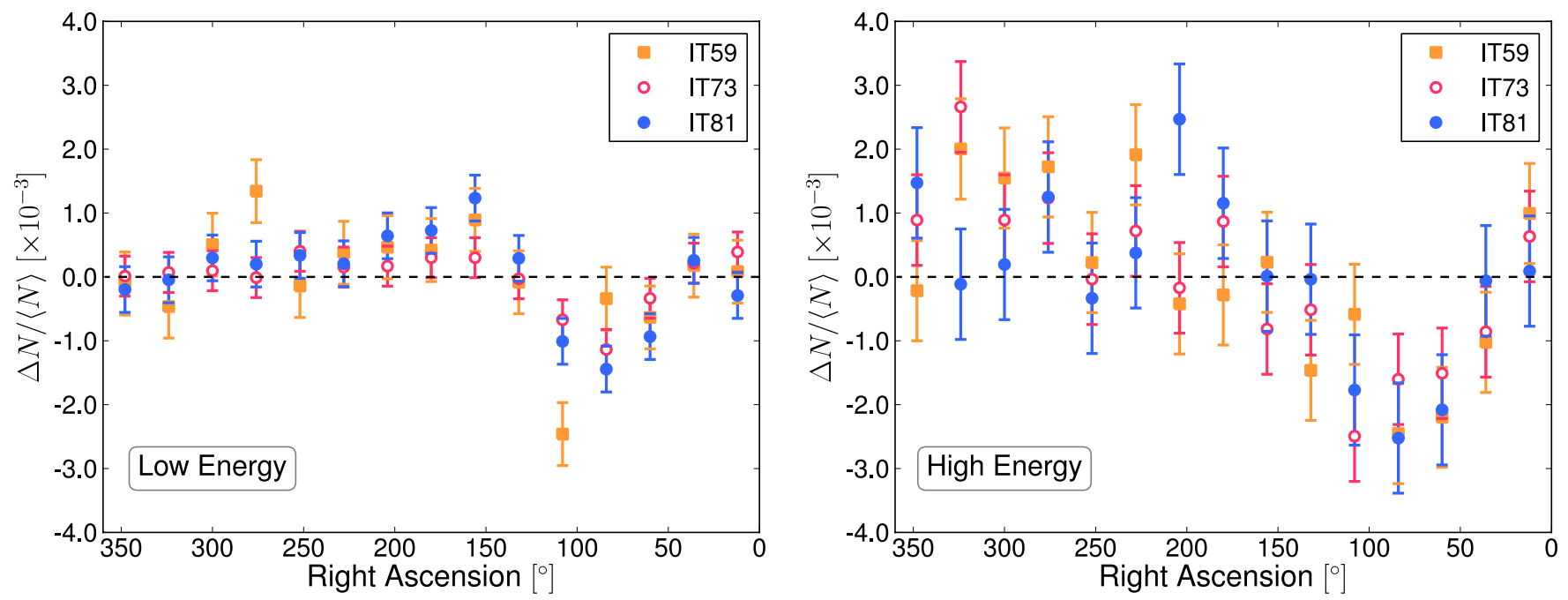

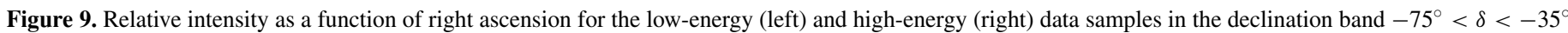
for the three detector configurations of IceTop considered in this work (IT59, IT73, and IT81). For clarity, only statistical error bars are shown.

(A color version of this figure is available in the online journal.) 


\section{CONCLUSIONS}

A study of cosmic-ray arrival directions with IceTop at two different median energies, $400 \mathrm{TeV}$ and $2 \mathrm{PeV}$, shows significant anisotropy in both sets. The skymap is dominated by a single deficit region with an angular size of about $30^{\circ}$. The skymap at $400 \mathrm{TeV}$ is similar to a skymap of comparable median energy obtained from cosmic rays in IceCube (Abbasi et al. 2012). IceTop data show that this anisotropy persists to $2 \mathrm{PeV}$.

The anisotropy in the southern sky at $400 \mathrm{TeV}$ and $2 \mathrm{PeV}$ is different in shape and amplitude from what is observed at $20 \mathrm{TeV}$. In the northern hemisphere, the EAS-TOP experiment has also recently found indications for an increasing amplitude and a change of phase between $100 \mathrm{TeV}$ and $400 \mathrm{TeV}$ in a harmonic analysis in right ascension that considers the first and second harmonic (Aglietta et al. 2009). The IceTop anisotropy is not well described by a sum of a dipole and a quadrupole moment, so the results cannot be directly compared. However, both northern and southern hemisphere data seem to show qualitatively similar trends.

Although these results do not provide conclusive evidence for any particular model, they lend support to scenarios where the large-scale anisotropy is a superposition of the flux from a few nearby sources. The sparse spatial distribution and the different ages of nearby supernova remnants are expected to lead to a bumpy structure in the amplitude and sudden changes in the phase of the anisotropy as a function of energy (Blasi \& Amato 2012). Unfortunately, this energy dependence is dominated by details such as the geometry of the Galaxy, the location, age and injection spectrum of the sources, and the energy dependence of the cosmic-ray diffusion coefficient. While the predicted strength of the amplitude has the correct order of magnitude, further quantitative predictions are not possible at this point. In addition, in their simplest form, these models predict a dipolar anisotropy, whereas in most cases, the observed anisotropy cannot be described as a simple dipole, which also means that "amplitude" and "phase" are not well defined.

It was recently pointed out that an existing dipolar flux in addition to cosmic-ray propagation in turbulent magnetic fields close to Earth can explain the appearance of small-scale structure (Giacinti \& Sigl 2012). For cosmic rays with energies from $\mathrm{TeV}$ to $\mathrm{PeV}$, the relevant distance scale is a few tens of parsecs, so the observed anisotropy at these energies is indicative of the turbulent Galactic magnetic field within this distance from Earth. The model predicts that the anisotropy is energy dependent, but again, due to our poor knowledge of interstellar magnetic fields, it cannot provide more quantitative predictions that can be tested with data. A detailed measurement of the anisotropy might lead to a better understanding of these fields.

The observation of cosmic-ray anisotropy with IceTop opens up new possibilities for future studies that go beyond mapping the arrival direction distribution as a function of energy. IceTop is designed to measure the energy spectrum and the chemical composition of the cosmic-ray flux above several hundred $\mathrm{TeV}$, and these capabilities allow for additional studies of the anisotropy. For one of the excess regions observed in the $10 \mathrm{TeV}$ skymap, the Milagro experiment has reported a different energy spectrum than the isotropic cosmic-ray flux (Abdo et al. 2008). With data from IceTop, studies of the energy spectrum and composition of the cosmic-ray flux in distinct regions of the southern sky can be performed.
IceTop is now in stable running mode in its complete configuration of 81 stations. In two years, the size of the cosmicray data set available for anisotropy studies will be more than twice what was used in the analysis presented in this paper. Eventually, it will be possible to extend the analysis of cosmicray anisotropy to higher energies.

Some of the results in this paper have been derived using the HEALPix (Gorski et al. 2005) software libraries.

We acknowledge the support from the following agencies: US National Science Foundation-Office of Polar Programs, US National Science Foundation-Physics Division, University of Wisconsin Alumni Research Foundation, the Grid Laboratory Of Wisconsin (GLOW) grid infrastructure at the University of Wisconsin-Madison, the Open Science Grid (OSG) grid infrastructure; US Department of Energy, and National Energy Research Scientific Computing Center, the Louisiana Optical Network Initiative (LONI) grid computing resources; National Science and Engineering Research Council of Canada; Swedish Research Council, Swedish Polar Research Secretariat, Swedish National Infrastructure for Computing (SNIC), and Knut and Alice Wallenberg Foundation, Sweden; German Ministry for Education and Research (BMBF), Deutsche Forschungsgemeinschaft (DFG), Research Department of Plasmas with Complex Interactions (Bochum), Germany; Fund for Scientific Research (FNRS-FWO), FWO Odysseus programme, Flanders Institute to encourage scientific and technological research in industry (IWT), Belgian Federal Science Policy Office (Belspo); University of Oxford, United Kingdom; Marsden Fund, New Zealand; Australian Research Council; Japan Society for Promotion of Science (JSPS); the Swiss National Science Foundation (SNSF), Switzerland.

\section{REFERENCES}

Abbasi, R., Abdou, Y., Abu-Zayyad, T., et al. 2010a, NIMPA, 618, 139 Abbasi, R., Abdou, Y., Abu-Zayyad, T., et al. 2010b, ApJL, 718, L194 Abbasi, R., Abdou, Y., Abu-Zayyad, T., et al. 2011, ApJ, 740, 16 Abbasi, R., Abdou, Y., Abu-Zayyad, T., et al. 2012, ApJ, 746, 33 Abbasi, R., Abdou, Y., Ackermann, M., et al. 2013, NIMPA, 700, 188 Abbasi, R., Ackermann, M., Adams, J., et al. 2009, NIMPA, 601, 294 Abdo, A. A., Allen, B., Aune, T., et al. 2008, PhRvL, 101, 221101 Abdo, A. A., Allen, B. T., Aune, T., et al. 2009, ApJ, 698, 2121 Aglietta, M., Alekseenko, V. V., Alessandro, B., et al. 2009, ApJL, 692, L130 Alexandreas, D. E., Berley, D., Biller, S., et al. 1993, NIMPA, 328, 570

Amenomori, M., Ayabe, S., Bi, X. J., et al. 2006, Sci, 314, 439

Amenomori, M., Ayabe, S., Cui, S. W., et al. 2005, ApJL, 626, L29

Blasi, P., \& Amato, E. 2012, JCAP, 01, 011

Erlykin, A. D., \& Wolfendale, A. 2006, APh, 25, 183

Gaisser, T. K. 2012, Aph, 35, 801

Giacinti, G., \& Sigl, G. 2012, PhRvL, 109, 071101

Gorski, K. M., Hivon, E., Banday, A. J., Wandelt, B. D., Hansen, F. K., Reinecke, M., \& Bartelmann, M. 2005, ApJ, 622, 759

Guillian, G., Hosaka, J., Ishihara, K., et al. 2007, PhRvD, 75, 062003

Han, J. L., Manchester, R. N., Lyne, A. G., Qiao, G. J., \& van Straten, W. 2006, ApJ, 642, 868

Heck, D., Knapp, J., Capdevielle, J. N., Schatz, G., \& Thouw, T. 1998, CORSIKA: A Monte Carlo Code to Simulate Extensive Air Showers, Technical Report FZKA 6019, Forschungszentrum Karlsruhe, http://www-ik.fzk.de/corsika/physics_description/corsika_phys.html Hörandel, J. R. 2003, APh, 19, 193

Li, T.-P., \& Ma, Y.-Q. 1983, ApJ, 272, 317

Munakata, K., Kiuchi, T., Yasue, S., et al. 1997, PhRvD, 56, 23

Pohl, M., \& Eichler, D. 2012, ApJ, submitted (arXiv:1208.5338)

Tilav, S., et al. 2009, in Proc. 31st ICRC, Łódź, Poland (arXiv:1001.0776)

Vernetto, S., et al. 2009, in Proc. 31st ICRC, Łódź, Poland (arXiv:0907.4615) 